\title{
Reactivity of 2-Ethoxyquinazolin- 4-yl hydrazine and its Use in Synthesis of Novel Quinazoline Derivatives of Antimicrobial Activity
}

\author{
Maher A El-Hashash, Sameh A Rizk, Fakhry A El-Bassiouny \\ Faculty of Science, Chemistry Department \\ Ain Shams University \\ Abbassia, Cairo - Egypt. \\ Khalid M Darwish (Corresponding author) \\ Science Faculty, Chemistry Department \\ Garyounis University \\ Benghazi-Libya \\ Tel: 0020113049976 Email: khaliddarwish1962@yahoo.com
}

Received: October 9, 2011 Accepted: October 24, 2011 Published: January 1, 2012

doi:10.5539/gjhs.v4n1p174 URL: http://dx.doi.org/10.5539/gjhs.v4n1p174

\begin{abstract}
The reactions of 2-ethoxy-4-hydrazinoquinazoline 2 with diethyl oxalate and ethyl chloroacetate gave 6-ethoxy-2H-[1,2,4] triazino [4,3-c] quinazoline-3,4-dione 3 and 6-ethoxy-2,3-dihydro-4H-[1,2,4] triazino [4, $3-c]$ quinazolin-4-one 4 respectively. A series of 5-ethoxy-2-X-[1,2,4] triazolo [1, 5-c] quinazolines 5a-d was also produced by reacting 2 with the acid chlorides namely: benzoyl, crotonyl, cinnamyl and 2-furoyl chlorides via Dimroth rearrangement. Also, 2 reacted with ethyl chloroformate giving 6. Condensation of 2 with acetone gave Schiff base 7, and with monosaccharides gave the sugar hydrazones 8a-e which was thereafter acetylated giving the corresponding 9a-e. Cyclization of 8a-e by iron(III) chloride gave triazoloquinazolines 10a-e acyclic $C$-nucleosides which, by acetylation, afforded $11 \mathrm{a}-\mathrm{e}$. All products were confirmed by elemental, IR, MS, and ${ }^{1} \mathrm{H}-\mathrm{NMR}$ analysis. Products 8-11 were chosen for biological screening test against gram (+ ive) and gram (- ive) bacteria.
\end{abstract}

Keywords: Iminamine, Triazoloquinazoline, Sugar Hydrazones, $C$-Nucleosides

\section{Introduction}

Quinazolines are a big family of heterocyclic compounds, which have shown broad variety of biological activity profiles, e. g. analgesic, antiinflammatory, antipyretic [1, 2], antimicrobial [3], anticonvulsant [4], anticancer [5], antitumoral [6], antihypertensive [7], antimalarial [8], diuretic [9], antidiabetic [10], antihistamine/sedative [11], antibiotic [12] and many others. Heterocycle-bearing $\mathrm{N}$-glycosides are well known to play a significant role as inhibitors. An example is the tetrazole-bearing N-glycosides used as SGLT2 inhibitors [13], where their hypoglycemic activity is tested in vivo by mice oral glucose tolerance test (OGTT). Moreover, sugar hydrazones exhibit remarkable biological activity [14]. Herein we report the synthesis of hydrazones of D-exoses and D-pentoses with 4-hydrazinoquinazoline and the screening of their antimicrobial potentials.

\section{Result and Discussion}

Recently, it was reported that 4-substituted-aminoquinazolines are exploited as potent antitumor compounds [15]. The 4-hydrazinoquinazolines resemble primary amines in being good substrates for aldehydes, ketones, alkyl and acid halides, anhydrides, etc. Therefore, they play a significant role in the synthesis of biologically active products [16]. The tautomeric behavior of hydrazinoquinazoline is used whenever necessary. For example, any necessary cyclization prior to product formation requires the presence of iminamine rather than hydrazine - form (Chart 1). 


\section{Chart 1: Tautomeric phenomenon of compound 2}<smiles>CCOc1nc(NN)c2ccccc2n1</smiles>

Hydrazino form<smiles>CCOc1nc2ccccc2/c(=N/N)[nH]1</smiles>

Iminamino form

Compound 2 reacted with diethyl oxalate and with ethyl chloroacetate in boiling ethanol to giving products 3 and 4 respectively (Scheme 1). The reaction possibly started with a nucleophilic attack of $\mathrm{NH} 2$ of hydrazine moiety on $\mathrm{C}=\mathrm{O}$ of the ester group through a tetrahedral mechanism intermediate to yield a fleeting acyl derivative followed by 1,3-tautomerism and ring closure via SN2 mechanism.<smiles>CCOc1nc2ccccc2c2n[nH]c(=O)c(=O)n12</smiles>

3

Scheme 1: synthetic pathway for compounds

3 and 4

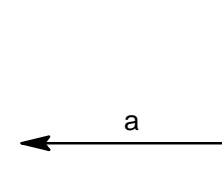<smiles>CCOc1nc2ccccc2c(=NN)[nH]1</smiles>

2<smiles>CCOC1=Nc2ccccc2C2=NNCC(=O)N12</smiles>

4

(a) EtOOC-COOEt / EtOH;

(b) $\mathrm{Cl}-\mathrm{CH}_{2} \mathrm{COOEt} / \mathrm{EtOH}$.

Similarly, compound 2 was reacted with acid halides namely: benzoyl, crotonyl, cinnamyl and furoyl chlorides in dry $\mathrm{CHCl}_{3}$ and $\mathrm{K}_{2} \mathrm{CO}_{3}$ giving the 2-acyl-1-(2-ethoxyquinazolin-4-yl)hydrazine derivatives, which tautomerized into the iminamide form upon heating and then underwent cyclization and Dimroth rearrangement affording the more stable derivatives 5a-d respectively (Scheme 2). Compound 2 was also reacted with ethyl chloroformate in dry pyridine affording derivative 6 .

\section{Scheme 2: synthetic pathway for compounds $\mathbf{5 a - d}$}

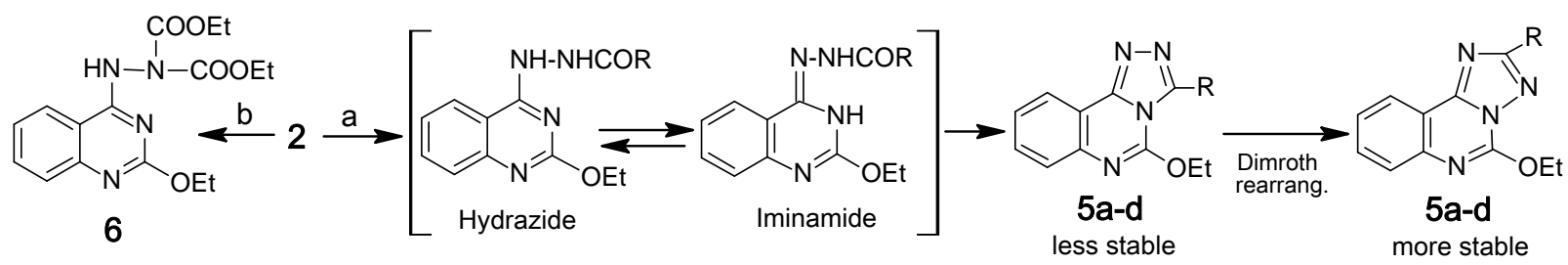

(a) $\mathrm{RCOCl} / \mathrm{CHCl}_{3} / \mathrm{K}_{2} \mathrm{CO}_{3}$; R: - $\mathrm{Ph}$; - $\mathrm{CH}=\mathrm{CH}-\mathrm{CH}_{3} ;-\mathrm{CH}=\mathrm{CH}-\mathrm{Ph}$; furan-2-yl;

(b) CICOOEt / pyridine.

Compound 2 was reacted with acetone affording derivative 7, whose mass spectrum showed a molecular ion peak at $m / z 244,246$ whereas the ${ }^{1} \mathrm{NMR}$ spectrum showed a singlet at $\delta 2.40 \mathrm{ppm}$ characteristic for $\mathrm{CH}_{3}$ groups of the hydrazone. A number of sugar hydrazones 8a-e were prepared by condensation of compound 2 with equimolar amounts of D-aldohexoses and D-aldopentoses namely: glucose, galactose, mannose, xylose and arabinose, respectively in boiling ethanol and drops of acetic acid as a catalyst (Scheme 3). Their IR spectra revealed characteristic absorption bands at $3459-3135 \mathrm{~cm}^{-1}$ attributed to $\mathrm{OH}$ and $\mathrm{NH}$ groups. Acetylation of these hydrazones $8 \mathrm{a}-\mathrm{e}$ by acetic anhydride in pyridine at room temperature afforded the corresponding per-acetyl products 9a-e, whose IR spectra revealed disappearance of the bands of $\mathrm{OH}$ groups and appearance of absorption bands in the carbonyl group frequency region at $1711-1725 \mathrm{~cm}^{-1}$ and $1673-1692 \mathrm{~cm}^{-1}$ due to the OAc and NAc groups, respectively. The ${ }^{1} \mathrm{H}-\mathrm{NMR}$ spectra showed signals corresponding to $O$-acetyl groups in addition to NAc groups; whereas no signals could be found for $\mathrm{NH}$ groups confirming that per- $O$ - and $\mathrm{N}$-acetylation had taken place. The 
spectra also confirmed the presence of the $\mathrm{HC}=\mathrm{N}$ proton as a doublet at $\delta 6.55-6.74 \mathrm{ppm}$ low field in addition to the rest of alditol-1-yl side chain.

Scheme 3: synthetic pathway for compounds 7 - 11

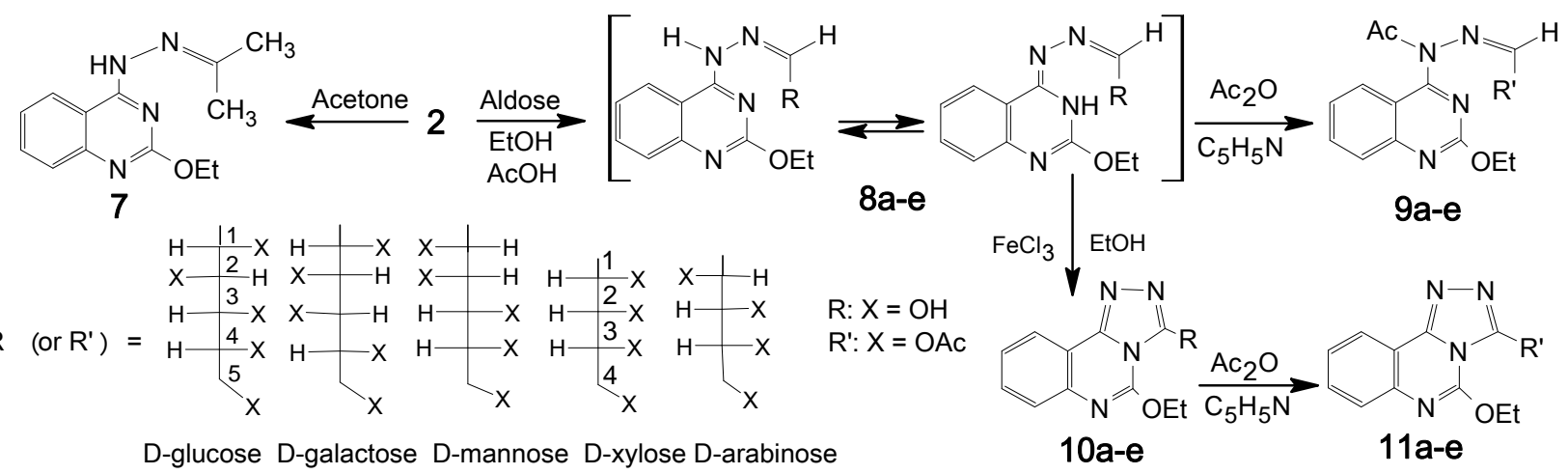

The mass spectral data of $9 \mathrm{e}$ showed a molecular ion peak at $\mathrm{m} / \mathrm{z} 546,548$ which agreed with the molecular formula $\mathrm{C}_{25} \mathrm{H}_{30} \mathrm{~N}_{4} \mathrm{O}_{10}$. The ion at $\mathrm{m} / z 215,217$ confirmed a loss of sugar residue from the molecular ion. The fragment at $m / z 175,177$ referred to quinazoline ring (Scheme 4).

Scheme 4: MS data interpretation of compound 9a

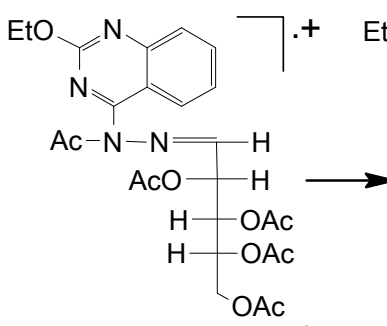

9 e $: m / z 546,548 \quad\left(\mathrm{M}^{+}\right)$

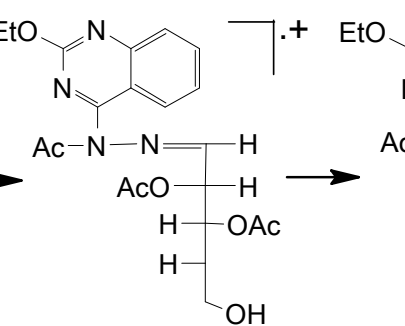

$m / z 504,506$

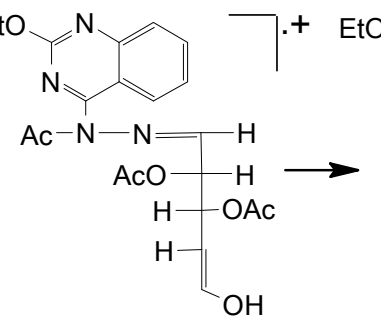<smiles>OC1=Nc2ccccc2[N+]=N1</smiles><smiles>CCOc1ncc2ccccc2n1</smiles>

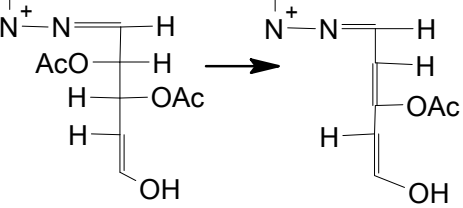<smiles>CCCCCC[I+]C</smiles>

$m / z 130,132$<smiles>C[O+]c1ncc2ccccc2n1</smiles>

$\mathrm{m} / \mathrm{z} 157,159$

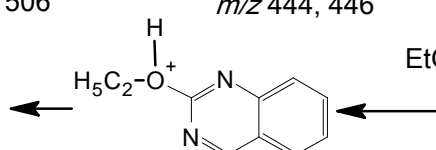

$\mathrm{m} / \mathrm{z} 175,177$

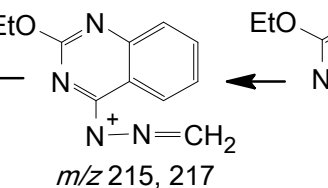
$m / z 341,343$

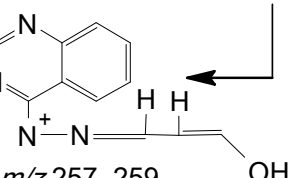

The oxidative cyclization of the hydrazones $8 \mathrm{a}-\mathrm{e}$ with ethanolic iron (III) chloride afforded the triazolo[4, 3-a]quinazolines 10a-e. The oxidation must have taken place by an electrophilic attack of the hard acid site of ferric chloride on the hardest basic site of sugar hydrazones 8a-e followed by an elimination of hydrogen chloride and formation of possibly a nitrilimine that undergoes 1,5-electrocyclization to give 10a-e. The IR spectra showed bands at 3240-3488 $\mathrm{cm}^{-1}(\mathrm{OH})$ and the mass spectral data of 10a showed a molecular ion peak at $\mathrm{m} / \mathrm{z} 364$ and 366 and an ion peak at $\mathrm{m} / \mathrm{z} 214$ and 216 presumably attributable to the triazoloquinazoline ring (Scheme 5). 
Scheme 5: MS data interpretation of compound 10a

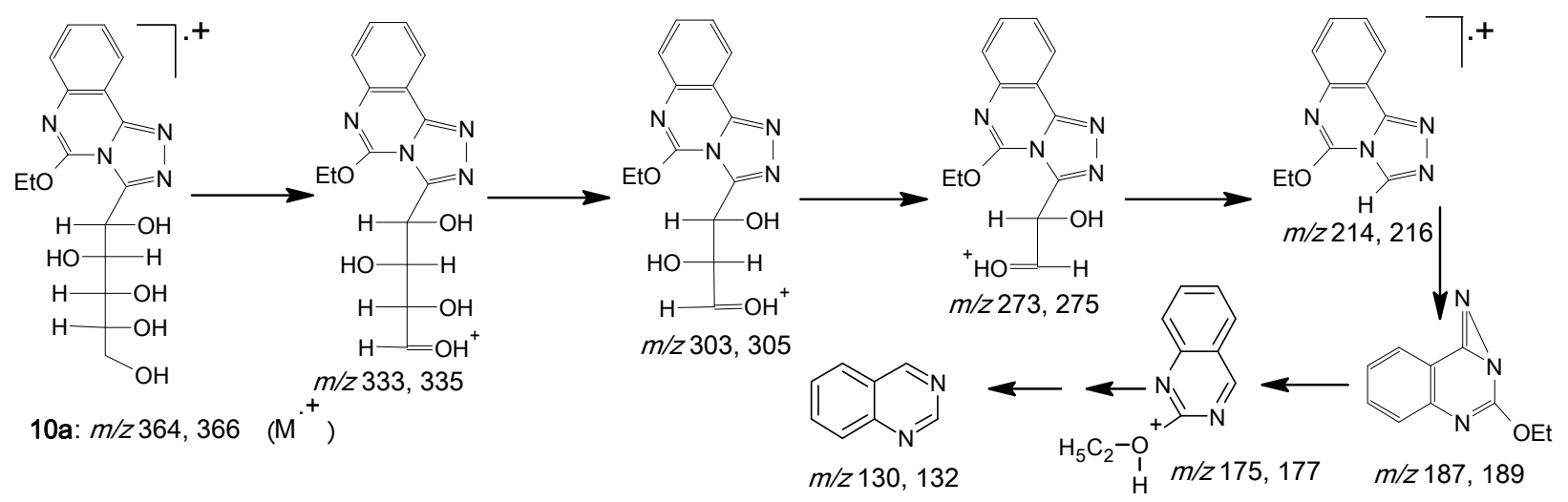

The ${ }^{1} \mathrm{H}-\mathrm{NMR}$ spectrum of compound $10 \mathrm{c}$ showed a doublet at low field at $\delta 5.22 \mathrm{ppm}$ assigned to $\mathrm{H}-1$, followed by the rest of the alditol-1-yl chain at higher field. The spectrum of $10 \mathrm{e}$ is similar, showing a doublet at low field at $\delta 5.03 \mathrm{ppm}$ for $\mathrm{H}-1$. Acetylation of $10 \mathrm{a}$-e by acetic anhydride in pyridine at room temperature gave polyacetoxyalkyl derivatives 11a-e, whose IR spectra showed only one absorption band in the $\mathrm{C}=\mathrm{O}$ frequency region (OA). The OAc groups were confirmed by the ${ }^{1} \mathrm{H}-\mathrm{NMR}$ spectra showing singlets at $\delta 2.03-2.19$. The doublets at $\delta$ 5.74-6.02 were attributed to $\mathrm{H}-1$. The mass spectra of products $11 \mathrm{~b}$ and $11 \mathrm{~d}$ showed molecular ion peaks at $\mathrm{m} / \mathrm{z} 574,576$ and 501, 503 (Scheme 6) which, on combination with the elemental analysis, led to the assignment of their molecular formulas $\mathrm{C}_{26} \mathrm{H}_{30} \mathrm{~N}_{4} \mathrm{O}_{11}$ and $\mathrm{C}_{23} \mathrm{H}_{26} \mathrm{~N}_{4} \mathrm{O}_{9}$ respectively. In addition, the characteristic fragment at $\mathrm{m} / \mathrm{z} 214,216$ was shown attributable to the triazoloquinazoline ring.

\section{Scheme 6: MS data interpretation of compound 11b}

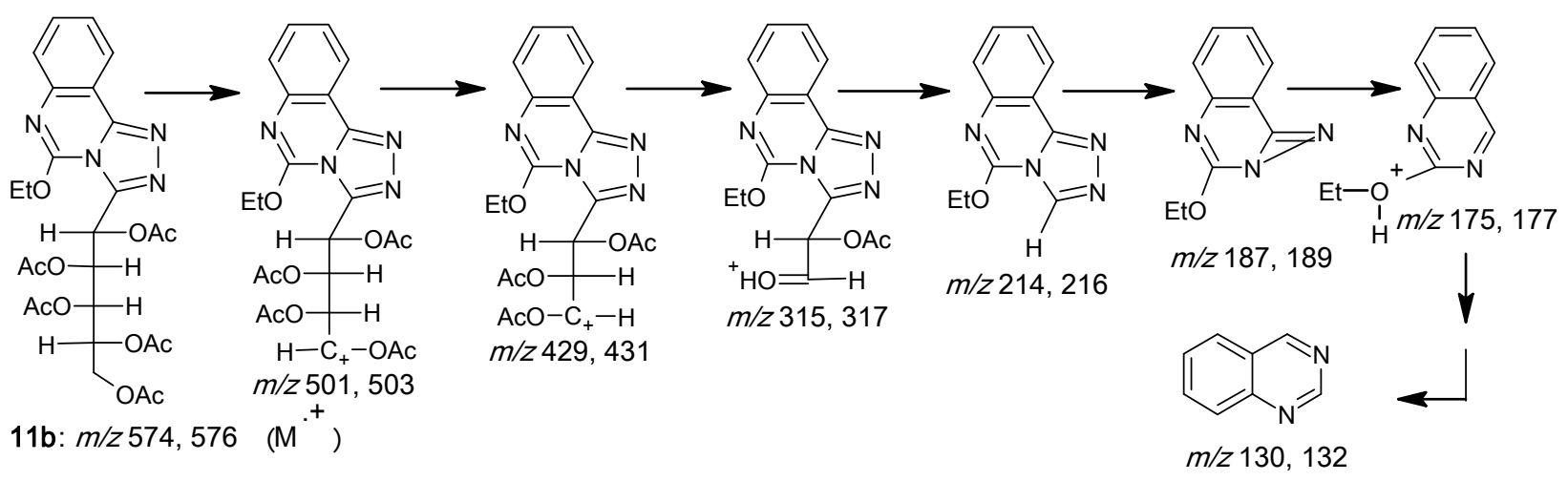

\subsection{Antimicrobial Activity}

All compounds were screened for their antimicrobial activity. Compounds 8-11 were tested against gram-positive bacteria Staphylococcus aureus, Streptobacillus moniliformis and Bacillus subtillis and gram-negative bacteria E. coli, Streptobacillus moniliformis and Pseudomonas acru-ginosa species applying the agar plate diffusion method. The screening results (Table 1) indicated that all the tested products exhibited antimicrobial activities against one or more type of bacteria. Almost all triazoloquinazoline products 10a-e and 11a-e showed more inhibition against the gram positive bacteria specially Streptobacillus than the gram negative one.

$<$ Table 1>

\subsection{Experimental}

All melting points recorded are uncorrected. The IR spectra were recorded on a Pye Unicam SP1200 spectrophotometer using $\mathrm{KBr}$ wafer technique. The ${ }^{1} \mathrm{H}-\mathrm{NMR}$ spectra were determined on a Varian FT-200 or Brucker AC-200 MHz instrument using TMS as an internal standard. Chemical shifts $(\delta)$ are expressed in ppm. The mass spectra were determined using MP model NS-5988 and Shimadzu single focusing mass spectrometer $(70 \mathrm{eV})$. All the solvents used were of HPLC/AnalaR grade. All reagents were used as received from Alfa Aesar.

Synthesis of 2-Ethoxyquinazolin-4-ylhydrazine 1. 
An emulsion of 4-chloro-2-ethoxyquinazoline $1(0.01 \mathrm{~mol})$ and hydrazine hydrate $(0.05 \mathrm{~mol})$ in benzene $(15 \mathrm{~mL})$ was stirred for $2 \mathrm{~h}$. The benzene-insoluble gum obtained was treated and washed with water, dried and crystallized from ethanol affording reddish brown crystals of product 2. Evaporation of solvent from the benzene-soluble fraction afforded a residue which was rinsed with water and air dried. Crystallization of the residue from absolute ethanol afforded product 2.

2-Ethoxyquinazolin-4-ylhydrazine 2.

Yield $68 \%$; m. p. $156-158^{\circ}$ C. Anal. for $\mathrm{C}_{10} \mathrm{H}_{12} \mathrm{~N}_{4} \mathrm{O}$ (M. wt. 204); Found: C, 58.86; H, 5.78; N, 27.45; Calcd: C, 58.82; H, 5.88; N, 27.45; IR v $\left(\mathrm{cm}^{-1}\right) 1620(\mathrm{C}=\mathrm{N}), 3160(\mathrm{NH}), 3250,3300\left(\mathrm{NH}_{2}\right) ; \mathrm{MS}: \mathrm{m} / z[\mathrm{M}+\mathrm{H}]^{+} 204 ;{ }^{1} \mathrm{H}-$ NMR $\left(\right.$ DMSO-d $\left._{6}\right) \delta 1.18(\mathrm{t}, 3 \mathrm{H}, \mathrm{CH} 3$ of ethoxy $J=7.4 \mathrm{~Hz}), 4.19\left(\mathrm{q}, 2 \mathrm{H}, \mathrm{CH}_{2}\right.$ of ethoxy $\left.J=7.4\right), 4.95$ (br. s, 3H, $\mathrm{NH}$ and $\left.\mathrm{NH}_{2}\right), 7.43-8.08(\mathrm{~m}, 4 \mathrm{H}, \mathrm{ArH})$.

6-ethoxy-2H-[1,2,4]triazino[4,3-c]quinazoline-3,4-dione 3.

A mixture of $2(2.04 \mathrm{~g}, 0.01 \mathrm{~mol})$ and diethyl oxalate $(1.46 \mathrm{~g}, 0.01 \mathrm{~mol})$ in boiling ethanol $(30 \mathrm{~mL})$ was heated under reflux for $10 \mathrm{~h}$. After cooling the separated solid was collected and recrystallized from THF to give white crystals of 3; m. p. 237-239 ${ }^{\circ} \mathrm{C}$; yield $58 \%$. Anal. for $\mathrm{C}_{12} \mathrm{H}_{10} \mathrm{~N}_{4} \mathrm{O}_{3}$ (M. wt. 258); Found: C, 54.21; H, 3.56; N, 21.89; Calcd: C, 55.81; H, 3.88; N, 21.71; IR v $\left(\mathrm{cm}^{-1}\right) 1680-1690(\mathrm{C}=\mathrm{O}), 3275(\mathrm{sec} \mathrm{NH}) ; \mathrm{MS}: m / z[\mathrm{M}+\mathrm{H}]^{+} 258$ $(77 \%) .{ }^{1} \mathrm{H}-\mathrm{NMR}\left(\mathrm{DMSO}_{6}\right) \delta 1.21\left(\mathrm{t}, 3 \mathrm{H}, \mathrm{CH}_{3}\right.$ of ethoxy $\left.J=7.4 \mathrm{~Hz}\right), 4.38\left(\mathrm{q}, 2 \mathrm{H}, \mathrm{CH}_{2}\right.$ of ethoxy $\left.J=7.4\right), 7.21$ -8.13(m, 5H, ArH nd NH), 10.20(s, 1H, NH, exchangeable).

6-ethoxy-2H-[1,2,4]triazino[4,3-c]quinazolin-3(4H)-one 4.

A mixture of $2(2.04 \mathrm{~g}, 0.01 \mathrm{~mol})$ and ethyl chloroacetate $(1.22 \mathrm{~g}, 0.01 \mathrm{~mol})$ in boiling ethanol $(35 \mathrm{~mL})$ was heated under reflux for $10 \mathrm{~h}$. The solid that separated after cooling was recrystallized from dioxane affording light brown crystals of 4 ; m. p. 213-216 ${ }^{\circ} \mathrm{C}$; yield $58 \%$. Anal. for $\mathrm{C}_{12} \mathrm{H}_{12} \mathrm{~N}_{4} \mathrm{O}_{2}$ (M. wt. 244); Found: C, 58.85; H, 4.53; N, 23.02; Calcd: C, 59.02; H, 4.92; N, 22.95; IR v $\left(\mathrm{cm}^{-1}\right) 1675(\mathrm{C}=\mathrm{O}), 2993(\mathrm{CH}), 3312(\mathrm{sec} \mathrm{NH})$; MS: $\mathrm{m} / z$ $[\mathrm{M}+\mathrm{H}]^{+} 244 ;{ }^{1} \mathrm{H}-\mathrm{NMR}\left(\mathrm{DMSO}_{6}\right) \delta 1.19\left(\mathrm{t}, 3 \mathrm{H}, \mathrm{CH}_{3}\right.$ of ethoxy $\left.J=7.4 \mathrm{~Hz}\right), 3.94,4.30\left(\mathrm{~m}, 2 \mathrm{H}, \mathrm{CH}_{2} \mathrm{CO}\right), 4.30(\mathrm{q}$, $2 \mathrm{H}, \mathrm{CH}_{2}$ of ethoxy $\left.J=7.4\right), 7.01-8.31(\mathrm{~m}, 5 \mathrm{H}, \mathrm{ArH}$ and $\mathrm{NH}$ of triazine), $9.96(\mathrm{~s}, 1 \mathrm{H}, \mathrm{NH}$, exchangeable).

Synthesis of 5-ethoxy-2-substituted[1,2,4]triazolo[1,5-c]quinazoline $5 a-d$.

To a solution of derivative $2(0.01 \mathrm{~mol})$ in dry chloroform $(100 \mathrm{~mL})$ containing anhydrous $\mathrm{K}_{2} \mathrm{CO}_{3}(1 \mathrm{~g})$ the acid chloride namely: benzoyl, crotonyl, cinnamyl and furoyl chlorides $(0.015 \mathrm{~mol})$ was added slowly. After the addition was complete, the mixture was stirred at room temperature for $30 \mathrm{~min}$ and then heated on a steam bath for $1 \mathrm{~h}$. The mixture was filtered, evaporated and the crude product was collected and crystallized from the proper solvent affording product $5 \mathrm{a}-\mathrm{d}$.

5-Ethoxy-2-phenyl[1,2,4]triazolo[1,5-c]quinazoline $5 a$.

Colorless needles from ethanol; m. p. $168-170^{\circ} \mathrm{C}$; yield $68 \%$. Anal. for $\mathrm{C}_{17} \mathrm{H}_{14} \mathrm{~N}_{4} \mathrm{O}$ (M. wt. 290); Found: C, 70.17; H, 4.91; N, 19.38; Calcd: C, 70.34; H, 4.83; N, 19.31; IR v $\left(\mathrm{cm}^{-1}\right) 1622(\mathrm{C}=\mathrm{N}), 3050(\mathrm{CH}) ; \mathrm{MS}: \mathrm{m} / z$ $[\mathrm{M}+\mathrm{H}]^{+} 290$ (32.2), 292 (12.3), 214 (100), 216 (23.5), 174 (43.8), 176 (8.2), 78 (13.2), 80 (0.3); ${ }^{1} \mathrm{H}-\mathrm{NMR}$ $\left(\mathrm{DMSO}_{6}\right) \delta 1.19\left(\mathrm{t}, 3 \mathrm{H}, \mathrm{CH}_{3}\right.$ of ethoxy $\left.J=7.4\right), 4.33\left(\mathrm{q}, 2 \mathrm{H}, \mathrm{CH}_{2}\right.$ of ethoxy $\left.J=7.4\right), 7.57-8.10(\mathrm{~m}, 5 \mathrm{H}$, phenyl), 7.62 - 8.65 (m, 4H, ArH).

5-Ethoxy-2-[(1E)-prop-1-en-1-yl][1,2,4]triazolo[1,5-c]quinazoline 5b.

Brown white crystals from ethanol; m. p. $223-225^{\circ} \mathrm{C}$; yield $71 \%$. Anal. for $\mathrm{C}_{14} \mathrm{H}_{14} \mathrm{~N}_{4} \mathrm{O}$ (M. wt. 254); Found: C, 66.28; H, 5.31; N, 22.23; Calcd: C, 66.14; H, 5.51; N, 22.05; IR v (cm $\left.{ }^{-1}\right) 1635(\mathrm{C}=\mathrm{N}), 3050(\mathrm{CH})$; MS: $\mathrm{m} / \mathrm{z}$ $[\mathrm{M}+\mathrm{H}]^{+} 254$ (48.2), $256(14.2), 174(100), 176(38.1) ;{ }^{1} \mathrm{H}-\mathrm{NMR}\left(\mathrm{DMSO}-\mathrm{d}_{6}\right) \delta 1.21\left(\mathrm{t}, 3 \mathrm{H}, \mathrm{CH}_{3}\right.$ of ethoxy $\left.J=7.4\right)$, $1.67\left(\mathrm{t}, 3 \mathrm{H}, \mathrm{CH}_{3}\right), 4.31\left(\mathrm{q}, 2 \mathrm{H}, \mathrm{CH}_{2}\right.$ of ethoxy $\left.J=7.4 \mathrm{~Hz}\right), 6.13\left(\mathrm{~d}, \mathrm{H}, \mathrm{CH}_{\text {trans }}\right), 6.70\left(\mathrm{~d}, \mathrm{H}, \mathrm{CH}_{\text {trans }}\right), 7.53-8.21(\mathrm{~m}$, $4 \mathrm{H}, \mathrm{ArH})$.

5-Ethoxy-2-[(E)-2-phenylethenyl][1,2,4]triazolo[1,5-c]quinazoline $5 c$.

Off-white crystals from ethanol; $153-155^{\circ} \mathrm{C}$; yield 62\%. Anal. for $\mathrm{C}_{19} \mathrm{H}_{16} \mathrm{~N}_{4} \mathrm{O}$ (M. wt. 316); Found: C, 72.84; $\mathrm{H}$, 5.19; N, 17.76; Calcd: C, 72.15; H, 5.06; N, 17.72; IR v $\left(\mathrm{cm}^{-1}\right) 1633(\mathrm{C}=\mathrm{N}), \mathrm{MS}: \mathrm{m} / \quad[\mathrm{M}+\mathrm{H}]^{+} 316(29.3), 318$ (12.8), 174 (100), 176 (41.1), 103 (12.7), $105(0.8) ;{ }^{1} \mathrm{H}-\mathrm{NMR}\left(\mathrm{DMSO}_{6}\right) \delta 1.2\left(\mathrm{t}, 3 \mathrm{H}, \mathrm{CH}_{3}\right.$ of ethoxy $\left.J=7.4 \mathrm{~Hz}\right)$, 4.38 (q, 2H, $\mathrm{CH}_{2}$ of ethoxy $\left.J=7.4\right), 7.09,7.48$ (2d, 2H, of two olefin protons), 7.4-7.6 (m, 5H, $\left.\mathrm{PhH}\right), 7.67-8.71$ (m, 4H, quinazoline).

5-Ethoxy-2-(furan-2-yl)[1,2,4]triazolo[1,5-c]quinazoline $5 d$.

White crystals from benzene; $163-164^{\circ} \mathrm{C}$; yield $68 \%$. Anal. for $\mathrm{C}_{15} \mathrm{H}_{12} \mathrm{~N}_{4} \mathrm{O}_{2}$ (M. wt. 280); Found: C, 64.38; H, 4.31; N, 20.07; Calcd: C, 64.29; H, 4.29; N, 20.00; IR v $\left(\mathrm{cm}^{-1}\right) 1619(\mathrm{C}=\mathrm{N}), \mathrm{MS}: \mathrm{m} / z[\mathrm{M}+\mathrm{H}]^{+} 280(33.2), 282$ 
(12.4), 174 (100), 176 (31.3), 60 (0.8), 61 (0.1); ${ }^{1} \mathrm{H}-\mathrm{NMR}\left(\mathrm{DMSO}_{6} \mathrm{~d}_{6}\right) \delta 1.2\left(\mathrm{t}, 3 \mathrm{H}, \mathrm{CH}_{3}\right.$ of ethoxy $\left.J=7.4 \mathrm{~Hz}\right), 4.2$ (q, $2 \mathrm{H}, \mathrm{CH}_{2}$ of ethoxy $\left.J=7.4\right), 6.79(\mathrm{dd}, 1 \mathrm{H}, J=3.6 \mathrm{~Hz}, J=1.6$, Furan-H), $7.29(\mathrm{~d}, 1 \mathrm{H}, J=4.4 \mathrm{~Hz}$, Furan-H), $7.76(\mathrm{~d}, 1 \mathrm{H}, J=1.6 \mathrm{~Hz}$, Furan-H), $7.5-8.2(\mathrm{~m}, 4 \mathrm{H}, \mathrm{ArH})$.

\section{1-(2-Ethoxyquinazolin-4-yl)-2-bis(ethoxycarbonyl) hydrazine}

6.

A mixture of $2(0.01 \mathrm{~mol})$ and ethyl chloroformate $(0.02 \mathrm{~mol})$ in dry pyridine $(20 \mathrm{~mL})$ was heated at boiling water bath for $4 \mathrm{~h}$. The solvent was evaporated under vacuum, the residue was cooled and crystallized from ethanol giving colorless needles of 6; m.p. $123-125^{\circ} \mathrm{C}$; yield $52 \%$. Anal. $\mathrm{C}_{16} \mathrm{H}_{20} \mathrm{~N}_{4} \mathrm{O}_{5}$ (M.wt. 348); Found: C, 55.27; H, 5.83; N, 16.08; Calcd: C, 55.17; H, 5.75; N, 16.09; IR v ( $\left.\mathrm{cm}^{-1}\right) 1250(\mathrm{C}-\mathrm{O}), 1622(\mathrm{C}=\mathrm{N}), 1731(\mathrm{C}=\mathrm{O})$, 2986 (C-H); MS: $m / z$ [M+H] 348 (31.5), 350 (14.1), 275 (2.8), 277 (13.2), 187 (0.8), 189 (0.2), 174 (100), 176 (39.4), 74 (0.7), 75(0.1); ${ }^{1} \mathrm{H}-\mathrm{NMR}\left(\mathrm{DMSO}_{6}\right) \delta 1.11-1.25$ (t, $9 \mathrm{H}, 3 \mathrm{CH}_{3}$ of ethoxy), 4.15-4.25(q, $6 \mathrm{H}, 3 \mathrm{CH}_{2}$ of ethoxy), 8.5-8.8(m, 4H, ArH), 10.05(s, 1H,NH).

\section{2-ethoxy-4-hydrazinoquinazoline Acetone hydrazone 7.}

A solution of crude $2(0.01 \mathrm{~mol})$ in acetone was left to stand for several days when the solvent had evaporated to give a solid from which the hydrazone $7(80 \%)$ was isolated by chromatography on silica gel $(30 \mathrm{~g}, 2.5 \%$ absolute ethanol-chloroform). Crystallization from hexane gave product 7 as colorless solid that turned deep yellow on exposure to light and air; m. p. $115-116^{\circ} \mathrm{C}$; yield $85 \%$. Anal. for $\mathrm{C}_{13} \mathrm{H}_{16} \mathrm{~N}_{4} \mathrm{O}$ (M. wt. 244); Found: C, 63.98; H, 6.61; N, 22.95; Calcd: C, 63.93; H, 6.56; N, 22.95; IR v $\left(\mathrm{cm}^{-1}\right) 1634(\mathrm{C}=\mathrm{N}), 2993(\mathrm{CH}), 3243(\mathrm{sec}$ NH); MS: $m / z[\mathrm{M}+\mathrm{H}]^{+} 244$ (33.1), 246 (12.6), 215 (100), 217 (19.6), 174 (55.8), 176 (1.2); ${ }^{1} \mathrm{H}-\mathrm{NMR}\left(\mathrm{DMSO}-\mathrm{d}_{6}\right)$ $\delta 1.13\left(\mathrm{t}, 3 \mathrm{H}, \mathrm{CH}_{3}\right.$ of ethoxy $\left.J=7.4\right), 2.4\left(3 \mathrm{H}, \mathrm{s},-\mathrm{N}=\mathrm{C}_{-} \mathrm{CH}_{3}\right), 4.23\left(\mathrm{q}, 2 \mathrm{H}, \mathrm{CH}_{2}\right.$ of ethoxy $\left.J=7.4\right), 7.1-8.3(\mathrm{~m}, 4 \mathrm{H}$, ArH), 8.3(br. s,1H, NH).

General procedure for the synthesis of sugar (2-ethoxyquinazolin-4-yl) hydrazones 8a-e.

To a suspension of 2-Ethoxy-4-hydrazinoquinazoline $2(0.01 \mathrm{~mol})$ in ethanol $(30 \mathrm{ml})$, was added a solution of selected sugar (D-glucose, D-galactose, D-mannose, D-xylose and D-arabinose $(0.01 \mathrm{~mol}))$ in water $(10 \mathrm{ml})$ and few drops of glacial acetic acid. The mixture was heated under reflux until reaction was judged complete by TLC (2-6 h). The solid product formed upon cooling was filtered off, washed with the minimum amount of ethanol, dried and finally crystallized from ethanol to afford the corresponding hydrazones 8a-e.

2-ethoxy-4-hydrazinoquinazoline-D-glucose hydrazone $\quad 8 \mathrm{a}$.

Yield $62 \%$ (from ethanol); m. p. $212-214{ }^{\circ} \mathrm{C}$; Anal. for $\mathrm{C}_{16} \mathrm{H}_{22} \mathrm{~N}_{4} \mathrm{O}_{6}$ (M. wt. 366); Found: C, 52.66; H, 6.16; N, 15.41; Calcd: C, 52.46; H, 6.01; N, 15.30; IR v $\left(\mathrm{cm}^{-1}\right) 1615(\mathrm{C}=\mathrm{N}), 3225-3417(\mathrm{OH}, \mathrm{NH}) ; \mathrm{MS}: \mathrm{m} / z[\mathrm{M}+\mathrm{H}]^{+}$ $366 ;{ }^{1} \mathrm{H}-\mathrm{NMR}\left(\mathrm{DMSO}-\mathrm{d}_{6}\right) \delta 1.17\left(\mathrm{t}, 3 \mathrm{H}, \mathrm{CH}_{3}\right.$ of ethoxy $J=7.4$ ), 4.17 (q, $2 \mathrm{H}, \mathrm{CH}_{2}$ of ethoxy $\left.J=7.4\right), 7.1-8.3(\mathrm{~m}$, $4 \mathrm{H}, \mathrm{ArH}), 8.33$ (br. s, 1H, NH).

2-ethoxy-4-hydrazinoquinazoline-D-galactose hydrazone $8 \mathrm{~b}$.

Yield 88\% (from DMF/ethanol); m. p. 193-195 ${ }^{\circ} \mathrm{C}$; Anal. for $\mathrm{C}_{16} \mathrm{H}_{22} \mathrm{~N}_{4} \mathrm{O}_{6}$ (M. wt. 366); Found: C, 52.56; H, 6.23; N, 15.48; Calcd: C, 52.46; H, 6.01; N, 15.30; IR v $\left(\mathrm{cm}^{-1}\right) 1615(\mathrm{C}=\mathrm{N}), 3135-3391(\mathrm{OH}$ and NH); $\mathrm{MS}: \mathrm{m} / z$ $[\mathrm{M}+\mathrm{H}]^{+} 366 ;{ }^{1} \mathrm{H}-\mathrm{NMR}$ (DMSO-d $\left.\mathrm{d}_{6}\right) \delta 1.13\left(\mathrm{t}, 3 \mathrm{H}, \mathrm{CH}_{3}\right.$ of ethoxy $J=7.4 \mathrm{~Hz}$ ), 4.21 (q, $2 \mathrm{H}, \mathrm{CH}_{2}$ of ethoxy $J=7.4$ ), 7.3 - 8.2 (m, 4H, ArH), 8.37 (br. s, 1H, NH).

2-ethoxy-4-hydrazinoquinazoline-D-mannose hydrazone $8 \mathrm{c}$.

Yield 77\% (from DMF/ethanol); m. p. 222-224 ${ }^{\circ} \mathrm{C}$; Anal. for $\mathrm{C}_{16} \mathrm{H}_{22} \mathrm{~N}_{4} \mathrm{O}_{6}$ (M. wt. 366); Found: C, 52.42; H, 6.04; N, 15.37; Calcd: C, 52.46; H, 6.01; N, 15.30; IR v $\left(\mathrm{cm}^{-1}\right) 1618(\mathrm{C}=\mathrm{N}), 3232-3459(\mathrm{OH}$ and NH); MS: $\mathrm{m} / z$ $[\mathrm{M}+\mathrm{H}]^{+} 366 ;{ }^{1} \mathrm{H}-\mathrm{NMR}\left(\mathrm{DMSO}-\mathrm{d}_{6}\right) \delta 1.15\left(\mathrm{t}, 3 \mathrm{H}, \mathrm{CH}_{3}\right.$ of ethoxy $\left.J=7.4 \mathrm{~Hz}\right), 4.23\left(\mathrm{q}, 2 \mathrm{H}, \mathrm{CH}_{2}\right.$ of ethoxy $J=7.4$ ), $7.0-8.1$ (m, 4H, ArH), 8.29 (br. s, 1H, NH).

2-ethoxy-4-hydrazinoquinazoline-D-ribose hydrazone

$8 \mathrm{~d}$.

Yield 61\% (from DMF/ethanol); m. p. 217-219 ${ }^{\circ} \mathrm{C}$; Anal. for $\mathrm{C}_{15} \mathrm{H}_{20} \mathrm{~N}_{4} \mathrm{O}_{5}$ (M. wt. 336); Found: C, 53.68; H, 6.04; N, 16.57; Calcd: C, 53.57; H, 5.95; N, 16.67; IR $v\left(\mathrm{~cm}^{-1}\right) 1616(\mathrm{C}=\mathrm{N}), 3210-3439(\mathrm{OH}$ and NH); MS: $\mathrm{m} / z$ $[\mathrm{M}+\mathrm{H}]^{+} 336 ;{ }^{1} \mathrm{H}-\mathrm{NMR}\left(\mathrm{DMSO}_{-} \mathrm{d}_{6}\right) \delta 1.13(\mathrm{t}, 3 \mathrm{H}, \mathrm{CH} 3$ of ethoxy $J=7.4 \mathrm{~Hz}), 4.23\left(\mathrm{q}, 2 \mathrm{H}, \mathrm{CH}_{2}\right.$ of ethoxy $J=$ 7.4), 7.1- 8.2 (m, 4H, ArH), 8.52 (br. s, $1 \mathrm{H}, \mathrm{NH})$.

2-ethoxy-4-hydrazinoquinazoline-D-arabinose hydrazone $8 \mathrm{e}$.

Yield 63\% (from ethanol); m. p. 197-198 ${ }^{\circ} \mathrm{C}$; Anal. for $\mathrm{C}_{15} \mathrm{H}_{20} \mathrm{~N}_{4} \mathrm{O}_{5}$ (M. wt. 336); Found: C, 53.62; H, 5.98; N, 16.63; Calcd: C, 53.57; H, 5.95; N, 16.67; IR v $\left(\mathrm{cm}^{-1}\right) 1613(\mathrm{C}=\mathrm{N}), 3230-3414\left(\mathrm{OH}\right.$ and NH); MS: $\mathrm{m} / z[\mathrm{M}+\mathrm{H}]^{+}$ $336 ;{ }^{1} \mathrm{H}-\mathrm{NMR}\left(\mathrm{DMSO}_{6}\right) \delta 1.11\left(\mathrm{t}, 3 \mathrm{H}, \mathrm{CH}_{3}\right.$ of ethoxy $\left.J=7.4 \mathrm{~Hz}\right), 4.19\left(\mathrm{q}, 2 \mathrm{H}, \mathrm{CH}_{2}\right.$ of ethoxy $\left.J=7.4\right), 7.2-8.3$ (m, $4 \mathrm{H}, \mathrm{ArH}), 8.33$ (br. s, 1H, NH). 
Synthesis of per-O-acetylsugar [1-acetyl-1-(2-ethoxyquinazolin-4-yl)] hydrazones 9a-e.

A cold solution of $8 \mathrm{a}$-e $(0.02 \mathrm{~mol})$ in dry pyridine $(50 \mathrm{~mL})$ was treated with $\mathrm{Ac}_{2} \mathrm{O}(50 \mathrm{~mL})$. The mixture was kept overnight at room temperature, with occasional shaking, and then poured onto crushed ice, and the residue was collected by filtration, washed repeatedly with water, dried and recrystallized from ethanol affording product $9 \mathrm{a}-\mathrm{e}$.

\section{2,3,4,5,6-Penta-O-acetyl-D-glucose[1-acetyl-1-(2-ethoxyquinazolin-4-yl)] hydrazones 9a.}

Yield $58 \%$; m. p. 63 - $64{ }^{\circ} \mathrm{C}$; Anal. for $\mathrm{C}_{28} \mathrm{H}_{34} \mathrm{~N}_{4} \mathrm{O}_{12}$ (M. wt. 618); Found: C, 54.41; H, 5.56; N, 9.01; Calcd: C, 54.37; H, 5.50; N, 9.06; IR $v\left(\mathrm{~cm}^{-1}\right) 1608(\mathrm{C}=\mathrm{N}), 1673(\mathrm{NAc}), 1718(\mathrm{OAc}) ; \mathrm{MS}: \mathrm{m} / z[\mathrm{M}+\mathrm{H}]^{+} 618 ;{ }^{1} \mathrm{H}-\mathrm{NMR}$ $\left(\right.$ DMSO-d $\left._{6}\right) \delta 1.13\left(\mathrm{t}, 3 \mathrm{H}, \mathrm{CH}_{3}\right.$ of ethoxy $\left.J=7.4 \mathrm{~Hz}\right), 2.02,2.04,2.10(3 \mathrm{~s}, 15 \mathrm{H}, 5 \mathrm{OAc}), 2.50(\mathrm{~s}, 3 \mathrm{H}, \mathrm{NAc})$, 4.1(q, 2H, $\mathrm{CH}_{2}$ of ethoxy $\left.J=7.4\right), 4.15$ (dd, $\left.1 \mathrm{H}, \mathrm{H}-6^{\prime}\right), 4.26$ (dd, 1H, H-6), 5.02-5.10 (m, 1H, H-5), 5.44-5.55 (m, 2H, H-4, H-3), 5.62 (dd, 1H, H-2), 6.74 (d, 1H, H-1), 7.11-8.23 (m, 4H, ArH).

\section{2,3,4,5,6-Penta-O-acetyl-D-galactose[1-acetyl-1-(2-ethoxyquinazolin-4-yl)] hydrazones 9b.}

Yield $80 \%$; m. p. $158-160{ }^{\circ} \mathrm{C}$; Anal. for $\mathrm{C}_{28} \mathrm{H}_{34} \mathrm{~N}_{4} \mathrm{O}_{12}$ (M. wt. 618); Found: C, 54.43; H, 5.53; N, 9.03; Calcd: C, 54.37; H, 5.50; N, 9.06; IR $v\left(\mathrm{~cm}^{-1}\right) 1633(\mathrm{C}=\mathrm{N}), 1692(\mathrm{NAc}), 1722(\mathrm{OAc}) ; \mathrm{MS}: \mathrm{m} / z[\mathrm{M}+\mathrm{H}]^{+} 618 ;{ }^{1} \mathrm{H}-\mathrm{NMR}$ $\left(\right.$ DMSO-d $\left._{6}\right) \delta 1.13\left(\mathrm{t}, 3 \mathrm{H}, \mathrm{CH}_{3}\right.$ of ethoxy $\left.J=7.4 \mathrm{~Hz}\right), 1.96,1.99,2.02,2.03,2.08,2.09(5 \mathrm{~s}, 15 \mathrm{H}, 5 \mathrm{OAc}), 2.47(\mathrm{~s}$, $3 \mathrm{H}, \mathrm{NAc}$ ), 3.88(dd, $1 \mathrm{H}, \mathrm{H}-6$ '), 4.1(q, $2 \mathrm{H}, \mathrm{CH}_{2}$ of ethoxy $J=7.4$ ), 4.28 (dd, $\left.1 \mathrm{H}, \mathrm{H}-6\right), 5.38-5.88$ (m, 4H, H-5, H-4, $\mathrm{H}-3, \mathrm{H}-2), 6.55$ (d, 1H, H-1), 7.15- 8.33 (m, 4H, ArH).

2,3,4,5,6-Penta-O-acetyl-D-mannose[1-acetyl-1-(2-ethoxyquinazolin-4-yl)] hydrazones 9c.

Yield $63 \%$; m. p. 58 - $60{ }^{\circ} \mathrm{C}$; Anal. for $\mathrm{C}_{28} \mathrm{H}_{34} \mathrm{~N}_{4} \mathrm{O}_{12}$ (M. wt. 618); Found: C, 54.40; H, 5.51; N, 9.02; Calcd: C, 54.37; H, 5.50; N, 9.06; IR $v\left(\mathrm{~cm}^{-1}\right) 1615(\mathrm{C}=\mathrm{N}), 1682(\mathrm{NAc}), 1711(\mathrm{OAc}) ; \mathrm{MS}: \mathrm{m} / z[\mathrm{M}+\mathrm{H}]^{+} 618 ;{ }^{1} \mathrm{H}-\mathrm{NMR}$ $\left(\right.$ DMSO-d $\left._{6}\right) \delta 1.13\left(\mathrm{t}, 3 \mathrm{H}, \mathrm{CH}_{3}\right.$ of ethoxy $\left.J=7.4 \mathrm{~Hz}\right), 2.05,2.06,2.10(3 \mathrm{~s}, 15 \mathrm{H}, 5 \mathrm{OAc}), 2.52(\mathrm{~s}, 3 \mathrm{H}, \mathrm{NAc})$, 4.11(q, $2 \mathrm{H}, \mathrm{CH}_{2}$ of ethoxy $\left.J=7.4\right), 4.14$ (dd, $1 \mathrm{H}, \mathrm{H}-6$ '), 4.28 (dd, 1H, H-6), 5.22-5.40 (m, 1H, H-5), 5.42 (d, $1 \mathrm{H}$, H-4), 5.54 (dd, 1H, H-3), 5.66 (dd, 1H, H-2), 6.68 (d, 1H, H-1), 7.00 - 8.13 (m, 4H, ArH).

\section{2,3,4,5-Tetra-O-acetyl-D-ribose[1-acetyl-1-(2-ethoxyquinazolin-4-yl)] hydrazones 9d.}

Yield 52 \%; m. p. $92-93{ }^{\circ} \mathrm{C}$; Anal. for $\mathrm{C}_{25} \mathrm{H}_{30} \mathrm{~N}_{4} \mathrm{O}_{10}$ (M. wt. 546); Found: C, 54.97; H, 5.54; N, 10.29; Calcd: C, 54.94; H, 5.49; N, 10.26; IR v $\left(\mathrm{cm}^{-1}\right) 1619(\mathrm{C}=\mathrm{N}), 1682$ (NAc), $1725(\mathrm{OAc})$; MS: $m / z[\mathrm{M}+\mathrm{H}]^{+}$546; ${ }^{1} \mathrm{H}-\mathrm{NMR}$ $\left(\right.$ DMSO-d $\left._{6}\right) \delta 1.91,1.94,2.00,2.19(4 \mathrm{~s}, 12 \mathrm{H}, 4 \mathrm{OAc}), 1.13\left(\mathrm{t}, 3 \mathrm{H}, \mathrm{CH}_{3}\right.$ of ethoxy $\left.J=7.4 \mathrm{~Hz}\right), 2.51(\mathrm{~s}, 3 \mathrm{H}, \mathrm{NAc})$, 4.15 (q, $2 \mathrm{H}, \mathrm{CH}_{2}$ of ethoxy $J=7.4$ ), 4.22 (dd, $\left.1 \mathrm{H}, \mathrm{H}-5^{\prime}\right), 4.39$ (dd, 1H, H-5), 5.38 - 5.40 (m, 1H, H-4), 5.78 (dd, $1 \mathrm{H}, \mathrm{H}-3), 6.01$ (dd, 1H, H-2), 6.59 (d, 1H, H-1), 7.10- 8.32 (m, 4H, ArH).

\section{2,3,4,5-Tetra-O-acetyl-D-arabinose[1-acetyl-1-(2-ethoxyquinazolin-4-yl)] hydrazones 9e.}

Yield 67 \%; m. p. 108-111 ${ }^{\circ}$ C; Anal. for $\mathrm{C}_{25} \mathrm{H}_{30} \mathrm{~N}_{4} \mathrm{O}_{10}$ (M. wt. 546); Found: C, 54.95; H, 5.52; N, 10.27; Calcd: C, 54.94; H, 5.49; N, 10.26; IR v $\left(\mathrm{cm}^{-1}\right) 1610(\mathrm{C}=\mathrm{N}), 1682$ (NAc), 1715 (OAc); MS: $m / z[\mathrm{M}+\mathrm{H}]^{+} 546(42.9)$, 548 (14.3), 504 (10.5), 506 (3.4), 444 (25.0), 446 (8.2), 401 (13.3), 403 (4.4), 341 (17.6), 343 (5.8), 257 (40.5), 259 (13.4), 215 (100), 217 (33.3), 175 (16.2), 177 (5.3), 157 (2.8), 159 (0.9), 130 (58.3), 132 (0.1).

General method for preparing 1-(alditol-1-yl) - 5-ethoxy [1,2,4]triazolo[1,5-c] quinazoline 10a-e.

A $2 \mathrm{M}$ solution of iron (III) chloride in $\mathrm{EtOH}(2 \mathrm{~mL})$ was added dropwise to a boiling solution of 8a-e (0.01 mol) in ethanol $(50 \mathrm{~mL})$. Heating was continued for $10 \mathrm{~min}$ and the mixture was then kept overnight at room temperature. The product was filtered, washed repeatedly with water, air dried and recrystallized from EtOH affording product $10 \mathrm{a}-\mathrm{e}$.

1-(D-gluco-pentitol-1-yl)-5-ethoxy[1,2,4]triazolo[1,5-c] quinazoline $10 \mathrm{a}$.

Yield 90 \%; m. p. 85-87 ${ }^{\circ} \mathrm{C}$; Anal. for $\mathrm{C}_{16} \mathrm{H}_{20} \mathrm{~N}_{4} \mathrm{O}_{6}$ (M. wt. 364); Found: C, 52.79; H, 5.52; N,15.43; Calcd: C, 52.75; H, 5.49; N, 15.38; IR v $\left(\mathrm{cm}^{-1}\right) 1613(\mathrm{C}=\mathrm{N}), 3240-3454(\mathrm{OH}) ; \mathrm{MS}: m / z[\mathrm{M}+\mathrm{H}]^{+} 364(14.4), 366(4.8), 333$ (0.3), 335 (0.1), $303(0.6), 305(0.2), 273$ (0.6), 275 (0.2), 214 (100), $216(33.4), 187(59.8), 189(19.6), 175(6.2)$, $177(2.1), 157(2.5), 159(0.5), 130(63.4), 132(0.1)$.

1-(D-galacto-pentitol-1-yl)-5-ethoxy [1,2,4]triazolo[1,5-c] quinazoline $10 \mathrm{~b}$.

Yield $85 \%$; m. p. $80^{\circ} \mathrm{C}$; Anal. for $\mathrm{C}_{16} \mathrm{H}_{20} \mathrm{~N}_{4} \mathrm{O}_{6}$ (M. wt. 364); Found: C, 52.77; H, 5.56;N, 15.48; Calcd: C, 52.74; H, 5.49; N, 15.38; IR v $\left(\mathrm{cm}^{-1}\right) 1619(\mathrm{C}=\mathrm{N}), 3340-3450(\mathrm{OH})$;

1-(D-manno-pentitol-1-yl)-5-ethoxy[1,2,4]triazolo[1,5-c] quinazoline $10 \mathrm{c}$. 
Yield $75 \%$; m. p. $101-102^{\circ} \mathrm{C}$; Anal. for $\mathrm{C}_{16} \mathrm{H}_{20} \mathrm{~N}_{4} \mathrm{O}_{6}$ (M. wt. 364); Found: C, 52.82; H, 5.59; N, 15.53; Calcd: C, 52.74; H, 5.49; N, 15.38; IR v $\left(\mathrm{cm}^{-1}\right) 1615(\mathrm{C}=\mathrm{N}), 3290-3466(\mathrm{OH}) ;{ }^{1} \mathrm{H}-\mathrm{NMR}\left(\mathrm{DMSO}-\mathrm{d}_{6}\right) \delta$ 3.97-4.20 (m, 2H, H-5', H-5), 4.40 (dd, 1H, H-4), 4.62 (dd, 1H, H-3), 5.03 (t, 1H, H-2), 5.22 (d, 1H, H-1), 7.10- 8.32 (m, 4H, ArH). 1-(D-ribo-pentitol-1-yl)-5-ethoxy [1,2,4]triazolo[1,5-c] quinazoline $10 \mathrm{~d}$.

Yield $68 \%$; m. p. $128{ }^{\circ} \mathrm{C}$; Anal. for $\mathrm{C}_{15} \mathrm{H}_{18} \mathrm{~N}_{4} \mathrm{O}_{5}$ (M. wt. 334); Found: C, 53.92; H, 5.43; N, 16.81; Calcd: C, 53.89; H, 5.39; N, 16.77; IR v $\left(\mathrm{cm}^{-1}\right) 1623(\mathrm{C}=\mathrm{N}), 3310-3444(\mathrm{OH}) ; \mathrm{MS}: m / z[\mathrm{M}+\mathrm{H}]^{+} 334$ (21.1), $336(7.0)$, 303 (3.5), 305 (1.1), 273 (0.6), 275 (0.2), 214 (9.9), 216 (3.2), 187 (100), 189 (33.2), 175 (5.4), 177 (1.1), 157 (1.8), $159(0.3), 130(56.4), 132(0.1)$.

1-(D-arabino-pentitol-1-yl)-5-ethoxy[1,2,4]triazolo[1,5-c] quinazoline 10e.

Yield $63 \%$; m. p. $108{ }^{\circ} \mathrm{C}$; Anal. for $\mathrm{C}_{15} \mathrm{H}_{18} \mathrm{~N}_{4} \mathrm{O}_{5}$ (M. wt. 334); Found: C, 53.97; H, 5.46; N, 16.88; Calcd: C, 53.89; H, 5.39; N, 16.77; IR v $\left(\mathrm{cm}^{-1}\right) 1619(\mathrm{C}=\mathrm{N}), 3320-3480(\mathrm{OH}) ;{ }^{1} \mathrm{H}-\mathrm{NMR}\left(\mathrm{DMSO}^{-} \mathrm{d}_{6}\right) \delta$ 3.49-3.75 (m, 2H, H-4', H-4), 4.18-4.39 (m, 2H, H-3, H-2), 5.03 (d, 1H, H-1), 7.1-8.3 (m, 4H, ArH).

Preparation of 1-(penta-O-acetylsugar-1-yl)-5-ethoxy-1,2,4-triazolo[1,5-c] quinazoline 11a-e

A cold solution of 10a-e $(0.002 \mathrm{~mol})$ in dry pyridine $(10 \mathrm{~mL})$ was treated with $\mathrm{Ac}_{2} \mathrm{O}(6 \mathrm{~mL})$, and the mixture was kept overnight at room temperature, with occasional shaking, and then poured onto crushed ice, and the residue was collected by filtration, washed repeatedly with water, dried and recrystallized from ethanol affording product $11 \mathrm{a}-\mathrm{e}$.

1-(1,2,3,4,5-penta-O-acetyl-D-glucopentitol-1-yl)-5-ethoxy-1,2,4-triazolo[1,5-c] quinazoline $11 \mathrm{a}$.

Yield $78 \%$; m. p. $71{ }^{\circ} \mathrm{C}$; Anal. for $\mathrm{C}_{26} \mathrm{H}_{30} \mathrm{~N}_{4} \mathrm{O}_{11}$ (M. wt. 574); Found: C, 54.42; H, 5.26; N, 9.83; Calcd: C, 54.36; H, 5.23; N, 9.76; IR v $\left(\mathrm{cm}^{-1}\right) 1650(\mathrm{C}=\mathrm{N}), 1725(\mathrm{OAc}) ;{ }^{1} \mathrm{H}-\mathrm{NMR}\left(\mathrm{DMSO}^{-} \mathrm{d}_{6}\right) \delta 1.99,2.01,2.03(3 \mathrm{~s}, 12 \mathrm{H}$, 4OAc), 3.98 (dd, 1H, H-5'), 4.30 (dd, 1H, H-5), 5.30-5.52 (m, 3H, H-4, H-3, H-2), 5.86 (d, 1H, H-1), 7.26-8.30 (m, $4 \mathrm{H}, \mathrm{ArH})$.

\section{1-(1,2,3,4,5-penta-O-acetyl-D-galactopentitol-1-yl)-5-ethoxy-1,2,4-triazolo[1,5-c] quinazoline 11b.}

Yield $81 \%$; m. p. $66{ }^{\circ} \mathrm{C}$; Anal. for $\mathrm{C}_{26} \mathrm{H}_{30} \mathrm{~N}_{4} \mathrm{O}_{11}$ (M. wt. 574); Found: C, 54.39; H, 5.24; N, 9.79; Calcd: C, 54.36; H, 5.23; N, 9.76; IR v $\left(\mathrm{cm}^{-1}\right)$ 1619(C=N), 1719(OAc); MS: $m / z[\mathrm{M}+\mathrm{H}]^{+} 574$ (19.3), 576 (6.3), 501 (27.4), 503(9.1), 429(31.7), 431 (10.5), 315 (9.5), 317 (3.2), 214 (100), 216 (33.3), 187 (59.8), 189 (19.9), 175(5.4), 177 (2.1), 157 (1.3), $159(0.2), 130$ (48.4), $132(0.1)$.

1-(1,2,3,4,5-penta-O-acetyl-D-mannopentitol-1-yl)-5-ethoxy-1,2,4-triazolo[1,5-c]quinazoline 11c.

Yield $63 \%$; m. p. $76^{\circ} \mathrm{C}$; Anal. for $\mathrm{C}_{26} \mathrm{H}_{30} \mathrm{~N}_{4} \mathrm{O}_{11}$ (M. wt. 574); Found: C, 54.41; H, 5.27;N, 9.81; Calcd: C, 54.36; $\mathrm{H}, 5.23$; N, 9.76; IR $v\left(\mathrm{~cm}^{-1}\right) 1639(\mathrm{C}=\mathrm{N}), 1745(\mathrm{OAc}) ;{ }^{1} \mathrm{H}-\mathrm{NMR}\left(\mathrm{DMSO}_{\mathrm{d}}\right)$ ) $81.96,1.99,2.03,2.08(4 \mathrm{~s}, 12 \mathrm{H}$, 4OAc), $3.90\left(\mathrm{dd}, 1 \mathrm{H}, \mathrm{H}-5^{\prime}\right), 4.32$ (dd, 1H, H-5), 5.30-5.53 (m, 3H, H-4, H-3, H-2), 5.76 (d, 1H, H-1), 7.15-8.31(m, 4H, ArH).

1-(1,2,3,4-tetra-O-acetyl-D-ribopentitol-1-yl)-5-ethoxy-1,2,4-triazolo[1,5-c] quinazoline $11 \mathrm{~d}$.

Yield $58 \%$; m. p. $71{ }^{\circ} \mathrm{C}$; Anal. for $\mathrm{C}_{23} \mathrm{H}_{26} \mathrm{~N}_{4} \mathrm{O}_{9}$ (M. wt. 502); Found: C, 54.94; H, 5.22; N, 11.14; Calcd: C, 54.98; H, 5.18; N, 11.16; IR v $\left(\mathrm{cm}^{-1}\right) 1629(\mathrm{C}=\mathrm{N}), 1731(\mathrm{C}=\mathrm{O})$; MS: $m / z[\mathrm{M}+\mathrm{H}]^{+} 502(21.3), 504(7.1), 430$ (17.6), 432 (5.8), 358 (21.4), 360 (7.1), 286(16.9), 288(5.6), 214 (100), 216 (33.2), 187(8.4), 189(2.8), 175(5.8), 177(1.9), 157(1.6), $159(0.1), 130(59.3), 132(0.1) .{ }^{1} \mathrm{H}-\mathrm{NMR}\left(\mathrm{DMSO}_{6}\right) \mathrm{d} \delta 1.13\left(\mathrm{t}, 3 \mathrm{H}, \mathrm{CH}_{3}\right.$ of ethoxy $\left.\mathrm{J}=7.4\right)$, 2.03, 2.06, 2.08 (3s, 12H, 4OAc), 4.05 (dd, 2H, H-4', H-4), 4.35-4.55 (m, 1H, H-3), 5.56(dd, 1H, H-2), 5.74 (d, $1 \mathrm{H}, \mathrm{H}-1), 7.1-8.3(\mathrm{~m}, 4 \mathrm{H}, \mathrm{ArH})$.

1-(1,2,3,4-tetra-O-acetyl-D-arabinopentitol-1-yl)-5-ethoxy-1,2,4-triazolo[1,5-c] quinazoline 11e. Yield 67\%; m. p. $98^{\circ} \mathrm{C}$; Anal. for $\mathrm{C}_{23} \mathrm{H}_{26} \mathrm{~N}_{4} \mathrm{O}_{9}$ (M. wt. 502); Found: C, 54.92; H, 5.24; N, 11.19; Calcd: C, 54.98; H, 5.18; N, 11.16; IR v $\left(\mathrm{cm}^{-1}\right) 1618(\mathrm{C}=\mathrm{N}) ;{ }^{1} \mathrm{H}-\mathrm{NMR}\left(\mathrm{DMSO}_{\mathrm{d}}\right) \delta 1.13\left(\mathrm{t}, 3 \mathrm{H}, \mathrm{CH}_{3}\right.$ of ethoxy $\left.\mathrm{J}=7.4\right), 1.81,1.86,1.91,2.19$ (4s, 12H, 4 OAc), 4.22 (dd, 2H, H-4', H-4), 5.35 - 5.41 (m, 1H,H-3), 5.76 (dd, 1H, H-2), 6.02 (d, 1H, H-1), 7.17 - $8.32(\mathrm{~m}, 4 \mathrm{H}, \mathrm{ArH})$.

\section{Acknowledgement}

The author wishes to express his gratitude to the chemistry department of Ain-Shams University for providing the research assistance for carrying out the pilot project.

\section{Authors' Statement}

\section{Competing Interests}

The authors declare no conflict of interest. 


\section{References}

Alagar, V. \& Pathak, U. S. (2007). Synthesis and antihypertensive activity of novel 3-benzyl -2-substituted-3H-[1,2,4]triazolo[5,1-b]quinazolin-9-ones. Bioorg and Med. Chem., 15, 3457-3462. http://dx.doi.org/10.1016/j.bmc.

2007.03.007

Alagarsamy, V., Giridhar, R., \& Yadav, M. R. (2005). Synthesis and H1-antihistaminic activity of some novel 1-substituted-4-(3-methylphenyl)-1,2,4-triazolo[4,3-a]quinazolin-5(4H)-ones. Biol Pharm Bull., 28 (8), 1531-1534. http://dx.doi.org/10.1248/bpb.28.1531

Alagarsamy, V., Shankar, D., \& Solomon, V. R. (2006). Synthesis of some novel 2-mercapto-3- (substituted amino)-5,6,7,8-tetrahydro-3H-benzo[4,5]thieno[2,3-d]pyrimidin- 4 -ones as analgesic and anti-inflammatory agents. Arkivoc, 16, 149-159.

Chevalier, J., Mahamoud, A., Baitiche, M., et al. (2010). Quinazoline derivatives are efficient chemosensitizers of antibiotic activity in Enterobacter aerogenes, Klebsiella pneumoniae and Pseudo-monas aeruginosa resistant strains. J. Antimicrob. Agents, 36 (2), 64-168. http://dx.doi.org/10.1016/j.ijantimicag.2010.03.027

Deacon, C. F. \& Holst, J. J. (2010). Linagliptin, a xanthine-based dipeptidyl peptidase-4 inhibitor with an unusual profile for the treatment of type 2 diabetes. Expert Opin Investig Drugs, 19 (1), 133-140. http://dx.doi.org/10.1517/13543780903463862

El-Azab, A. S., Al-Omar, M. A., \& Abdel-Aziz, A. A., et al. (2010). Design, synthesis and biological evaluation of novel quinazoline derivatives as potential antitumor agents: molecular docking study. Eur J Med Chem., 45 (9), 4188-98. http://dx.doi.org/10.1016/j.ejmech.2010.06.013

Gao, Y. L., Zhao, G. L., Liu, W., et al. (2010). Design, synthesis and in vivo hypoglycemic activity of tetrazole-bearing N-glycosides as SGLT2 inhibitors. Ind. J. of Chem., 49 B, 1499-1508.

Ghorab, M. M., Ismail, Z. H., \& Abdalla, M. (2010). Synthesis and biological activities of some novel triazoloquinazolines and triazinoquinazolines ontaining benzenesulfonamide moieties. Arzneimittelforschung, 60 (2), 87-95. http://dx.doi.org/10.1055/s-0031-1296254

Guan, J., Zhang, Q., O'Neil, M., et al. (2005). Antimalarial activities of new pyrrolo [3,2-f]quinazoline-1, 3-diamine derivatives. Antimicrob Agents Chemother, 49 (12), 4928-4933. http://dx.doi.org/10.1128/AAC.49.12.4928-4933.2005

Khalil, A. A., AbdelHamide, S. G., Al-Obaid, A. M., et al. (2003). Substituted Quinazolines, Part 2. Synthesis and In-Vitro Anticancer Evaluation of New 2-Substituted Mercapto-3H-quinazoline Analogs. Arch. Pharm. Med. Chem., 2, 95-103. http://dx.doi.org/10.1002/ardp.200390011

Kundu, S. K., Mahindaratne, M. P. D., Quinrero, M. V., et al. (2008). One-pot reductive cyclization to antitumor quinazoline precursors. Arkivoc, 2, 33-42.

Laddha, S. S. \& Bhatnagar, S. P. (2009). Novel Fused quinazolinones: Further studies on the anti-convulsant activity of 1,2,9,11-tetrasubstituted-7H-thieno $\quad\left[2^{\prime}, 3^{\prime}: 4,5\right]$ pyrimido [6,1-b]-quinaz-olin-7-one and 1,3,10,12-tetrasubstituted-8H-pyrido[2',3':4,5]pyrimido[6,1-b]quinazolin-8-one. In proceedings of the 13th International Electronic Conference on Synthetic Organic Chemistry. [Online] Available: http://www.usc.es/con gresos/ecsoc/

Maarouf, A. R., El-Bendary, E. R., \& Goda, F. E. (2004). Synthesis and evaluation of some novel quinazolinone derivatives as diuretic agents. Arch Pharm (Weinheim), 337 (10), 527-532. http://dx.doi.org/10.1002/ardp.2004 00869

Mehta, S., Swarnkar, N., Vyas, M., et al. (2007). Synthesis and Characterization of Some Quinazoline Derivatives as Potential Antimicrobial Agents under Microwave Irradiation. Bull. Korean Chem. Soc., 28 (12), 2338-2342. http://dx.doi.org/10.5012/bkcs.2007.28.12.2338

Mohamed, M. S., Ibrahim, M. K., Alafify, A. M., et al. (2005). Synthesis and Anti-inflammatory Evaluation of some New Quinazoline Derivatives. Int. J. of Pharm., 1 (3), 261-266. http://dx.doi.org/10.3923/ijp.2005.261.266

Mosselhi, M. A., Abdallah, M. A., Metwally, N. H., et al. (2009). Synthesis, stucture and antimicrobial evaluation of new derivatives of theophylline sugar hydrazones. Arkivoc, 14, 53-63. 
Table 1. Antimicrobial activity

\begin{tabular}{|c|c|c|c|c|c|c|}
\hline \multirow{2}{*}{$\begin{array}{c}\text { Compd } \\
\text { No }\end{array}$} & \multicolumn{3}{|c|}{ Gram-positive Bacteria } & \multicolumn{3}{c|}{ Gram-negative Bacteria } \\
\cline { 2 - 8 } & $\begin{array}{c}\text { Staphy- } \\
\text { lococcussp }\end{array}$ & $\begin{array}{c}\text { Strepto- } \\
\text { bacillussp }\end{array}$ & $\begin{array}{c}\text { Bacillus- } \\
\text { subtillissp }\end{array}$ & $\begin{array}{c}\text { Esch- } \\
\text { colisp }\end{array}$ & $\begin{array}{c}\text { Strepto- } \\
\text { bacillusussp }\end{array}$ & $\begin{array}{c}\text { Pseudo- } \\
\text { monassp }\end{array}$ \\
\hline $8 \mathrm{a}$ & - & + & - & - & - & - \\
\hline $8 \mathrm{~b}$ & - & + & - & - & - & - \\
\hline $8 \mathrm{c}$ & - & + & + & - & - & - \\
\hline $8 \mathrm{~d}$ & - & + & - & - & - & - \\
\hline $8 \mathrm{e}$ & - & + & - & - & - & - \\
\hline $9 \mathrm{a}$ & - & + & + & - & + & - \\
\hline $9 \mathrm{~b}$ & - & + & + & + & - & - \\
\hline $9 \mathrm{c}$ & - & + & + & - & - & + \\
\hline $9 \mathrm{~d}$ & - & + & - & + & - & - \\
\hline $9 \mathrm{e}$ & - & + & + & + & - & + \\
\hline $10 \mathrm{a}$ & - & + & + & + & - & - \\
\hline $10 \mathrm{~b}$ & - & + & + & + & + & + \\
\hline $10 \mathrm{c}$ & - & ++ & ++ & + & + & + \\
\hline $10 \mathrm{~d}$ & + & +++ & ++ & + & - & - \\
\hline $10 \mathrm{e}$ & + & + & + & + & - & - \\
\hline $11 \mathrm{a}$ & - & +++ & ++ & + & + & + \\
\hline $11 \mathrm{~b}$ & - & ++ & + & + & - & - \\
\hline $11 \mathrm{c}$ & - & ++ & + & + & - & - \\
\hline $11 \mathrm{~d}$ & + & + & + & + & - & - \\
\hline $11 \mathrm{e}$ & - & ++ & + & + & - & - \\
\hline & & & & & & + \\
\hline
\end{tabular}

